\title{
Can new objects override attentional control settings?
}

\author{
CHARLES L. FOLK \\ Villanova University, Villanova, Pennsylvania \\ and \\ ROGER REMINGTON \\ NASA Ames Research Center, Moffett Field, California
}

\begin{abstract}
Previous research suggests that attentional capture by abrupt onsets is contingent on top-down attentional control settings. Four experiments addressed whether similar contingencies hold for capture elicited by the appearance of new perceptual objects. In a modified spatial cuing task, targets defined by abrupt onset or color were paired with distractors consisting of an abrupt brightening of an existing object or the abrupt appearance of a new object. In Experiments 1 and 2, when subjects searched for an onset target, both distractor types produced evidence of capture. When subjects searched for a color target, however, distractors produced no evidence of attentional capture, regardless of whether they consisted of a new perceptual object or not. Experiments 3-5 showed that the lack of distractor effects in the color-target condition cannot be accounted for by rapid recovery from capture. It was concluded that attentional capture by new objects is subject to top-down modulation by attentional control settings.
\end{abstract}

The interaction of bottom-up and top-down factors in the allocation of spatial attention is a key element of theories of visual selective attention. With respect to bottomup factors, considerable evidence suggests that salient stimulus events can exert a strong influence over the allocation of spatial attention (see Yantis, 1993, 1996, for reviews). For example, Yantis and his colleagues have shown that abrupt visual onsets can "capture" spatial attention. Specifically, when the target in a visual search task is abruptly onset among a variable number of characters whose identities are revealed by the removal of masking line segments, search slopes are substantially reduced. This holds even when the location of the target is uncorrelated with the location of the onset character across trials- that is, when there is no incentive to voluntarily search the onset character first (Yantis \& Jonides, 1984). These observations are consistent with the hypothesis that the onset character receives attentional priority independent of intention (but see Gibson, 1996, for a different interpretation). Similar effects have been obtained with the spatial cuing paradigm; an abruptly onset spatial precue with variable validity can produce costs and benefits in the detection or identification of a subsequent target. Again, this holds even when the location of the precue and target are uncorrelated (Folk, Remington, \& Johnston, 1992; Juola, Ko-

The authors thank Jan Theeuwes, Kyle Cave, and two anonymous reviewers for useful comments on a previous version of this manuscript. Correspondence should be addressed to C. L. Folk, Department of Psychology, Villanova University, Villanova, PA 19085 (e-mail: cfolk@ email.vill.edu).

-Accepted by previous editor, Myron L. Braunstein shino, \& Warner, 1995; Posner \& Cohen, 1984; Theeuwes, 1991).

In addition to onsets, there is evidence that irrelevant, salient discontinuities or "singletons" in feature properties such as color can also disrupt performance, presumably by capturing spatial attention (see, e.g., Theeuwes, 1992). Using a visual search task, Theeuwes (1992) found that when subjects were searching for a target defined as a shape singleton, the presence of an irrelevant color singleton disrupted performance relative to displays in which no irrelevant singleton was present. Moreover, this disruption effect was tied to the relative salience of the target and distractor singletons.

Although these studies clearly demonstrate the power of stimulus salience, it has nonetheless been repeatedly shown that these bottom-up effects can be modulated by top-down behavioral goals. For example, in the case of abrupt onsets, Yantis and Jonides (1990) found that when attention is focused at the target location by a $100 \%$ valid precue, subsequent abrupt onset characters no longer produce evidence of attentional capture. These results suggest that capture by onsets can be modulated by a top-down set for location (see also Juola et al., 1995; Theeuwes, 1991). In the case of feature singletons used by Theeuwes (1992), Bacon and Egeth (1994) have shown that the effects of an irrelevant singleton are eliminated when subjects are encouraged to establish a top-down attentional set for a specific feature property.

Additional evidence for the modulation of attentional capture by top-down factors comes from studies using a modified spatial cuing paradigm (Folk \& Remington, 1998; Folk et al., 1992; Folk, Remington, \& Wright, 1994; Gibson \& Kelsey, 1998). Folk et al. (1992, Experiment 3) 
had subjects search for a target that could appear in one of four boxes surrounding fixation. The target consisted of a single, abruptly onset character (onset target) appearing in one of the boxes, or one red character in one box and three white characters in each of the other boxes (color target). The onset-target condition was assumed to encourage a top-down attentional set for onset, and the color-target condition a top-down set for color. Target displays were preceded by a cue consisting of the abrupt onset of four small circles surrounding one box (onset cue) or a set of red circles surrounding one box and three sets of white circles surrounding the other three boxes (color cue). In all conditions, the location of the precue was uncorrelated with the location of the target (i.e., $25 \%$ valid, $75 \%$ invalid), so that subjects had no incentive to voluntarily shift attention to the cue.

With this design, Folk et al. (1992) found cue validity effects consistent with attentional capture only when targets and precues were both defined by onsets or were both defined by color. No cue validity effects were observed when onset (color) precues were paired with color (onset) targets. On the basis of these and similar results, Folk et al. (1992; Folk, Remington, \& Johnston, 1993) proposed the contingent involuntary orienting hypothesis, which states that all attentional capture is ultimately contingent on topdown attentional set.

It remains possible, however, that there exist stimulus properties that are so salient that they can override any existing top-down attentional set. One such property has recently been investigated by Yantis and Hillstrom (1994). They have argued that it is not abrupt onsets per se that produce capture, but rather the fact that abrupt onsets are typically correlated with the abrupt appearance of a new $o b$ ject. Using the visual search paradigm, they found that the appearance of a new object in the absence of an abrupt luminance change can produce attentional capture. Specifically, search slopes were significantly reduced when targets appeared as a new object defined in terms of discontinuities in retinal disparity, texture, or motion, independent of abrupt changes in luminance. Moreover, they found that irrelevant abrupt luminance change of an existing object did not produce evidence of capture. Thus, Yantis and Hillstrom proposed that abrupt onsets are neither necessary nor sufficient to capture attention. Rather, the formation of a new "object file" (Treisman, Kahneman, \& Burkell, 1983) is the functional requirement for attentional capture.

These new findings suggest that the abrupt appearance of a new object exerts more powerful stimulus-driven control over the allocation of spatial attention than abrupt onset alone. Given that our previous research on the topdown modulation of capture has focused on abrupt onsets that "highlight" existing objects rather than heralding new objects, it is possible that the appearance of a new object could override even a top-down attentional set for a feature property such as color. Such a result would support the notion that certain stimulus properties do indeed produce purely stimulus-driven shifts of attention and would clearly limit the generalizability of the contingent involuntary orienting hypothesis.
There is, in fact, some visual search evidence that appears to support the notion that new objects can override attentional set. Theeuwes (1994) presented subjects with fixation displays consisting of circles containing an asterisk symbol. Presentation of the target display was marked by a simultaneous change in color of one of the circles and the removal of all but one of the line segments of the asterisk in each circle. In the relevant condition, subjects were required to identify the orientation of the remaining line segment in the circle that changed color. Thus, the task encouraged a top-down attentional set for color. Performance in this baseline condition was compared with that in a condition in which the appearance of the color target was accompanied by the simultaneous presentation of an abruptly onset circle distractor containing a line segment at a location that had not previously contained a circle (i.e., the distractor circle was a new object). The presence of this abrupt onset (i.e., new object) distractor produced a significant cost in performance relative to the baseline condition, suggesting that the distractor captured attention even in the presence of a top-down attentional set for color.

The problem, however, is that it is difficult to interpret the costs associated with an irrelevant distractor relative to a "no-distractor" baseline. Folk and Remington (1998) have recently shown that irrelevant distractors can produce a "filtering cost" (Treisman et al., 1983) that is dissociable from actual shifts of spatial attention. Thus, the cost associated with the presence of a new object in Theeuwes's experiment may reflect a delay in attentional deployment associated with the presence of an additional visual "event" (i.e., the distractor) rather than a true shift of attention.

Theeuwes (1996) has argued against the "filtering cost" interpretation by showing that the identity of the character at the distractor location produces compatibility effects in response time (RT) to the target character. The presence of a compatibility effect associated with the character at the distractor location was taken as direct evidence that spatial attention was selectively allocated to the distractor location. This interpretation, however, assumes that distractor compatibility effects occur only when spatial attention is selectively allocated to the distractor location. Lavie (1995), however, has recently used the compatibility effect to show that identities of a small number of objects can be processed in parallel under conditions of low perceptual load. If one assumes that the distractor displays used by Theeuwes (1996) are coded as low-load displays consisting of only two "objects" (the target singleton and the distractor singleton), then the compatibility effects associated with Theeuwes's distractors may reflect parallel processing of these two objects rather than the selective allocation of spatial attention to the distractor.

The present experiments were designed to critically assess whether the appearance of a new object can produce an involuntary shift of spatial attention that occurs regardless of the top-down attentional set of the observer. The general design of the experiments was similar to the modified spatial cuing studies of Folk et al. (1992, Experiment 3). Subjects searched for targets defined by abrupt 


\section{TARGET DISPLAYS}

\section{Onset Target}

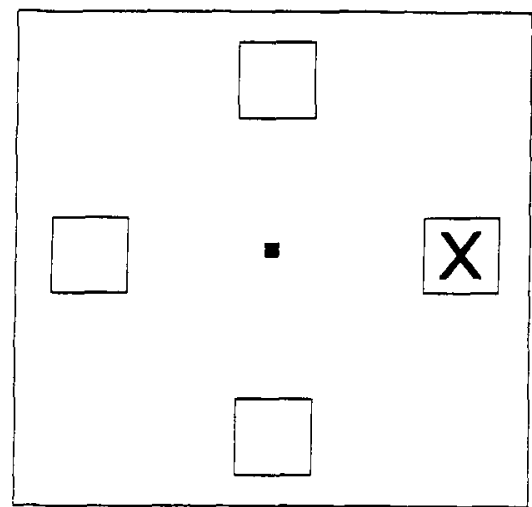

Color Target

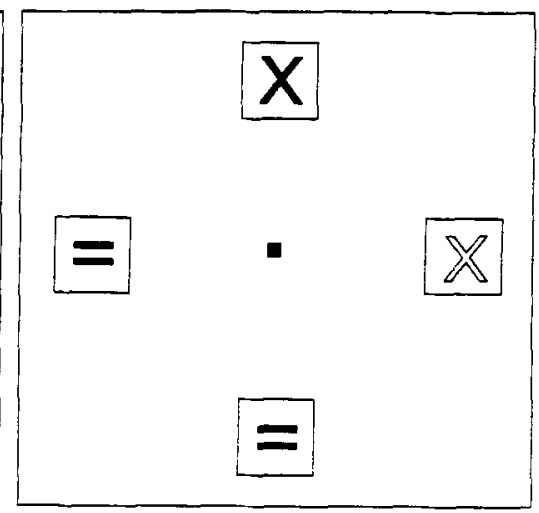

\section{DISTRACTOR DISPLAYS}

\section{Old-Object Distractor}

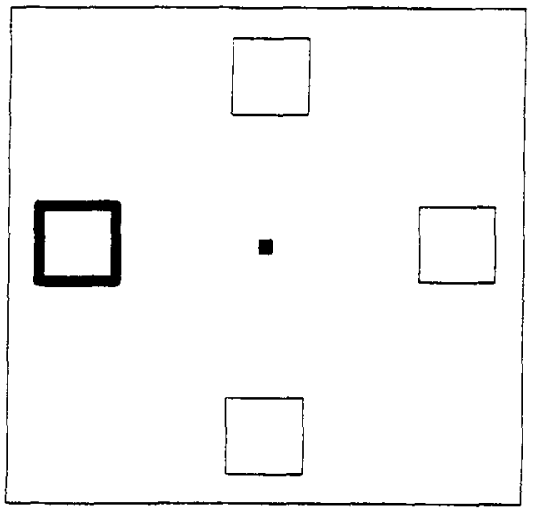

New-Object Distractor

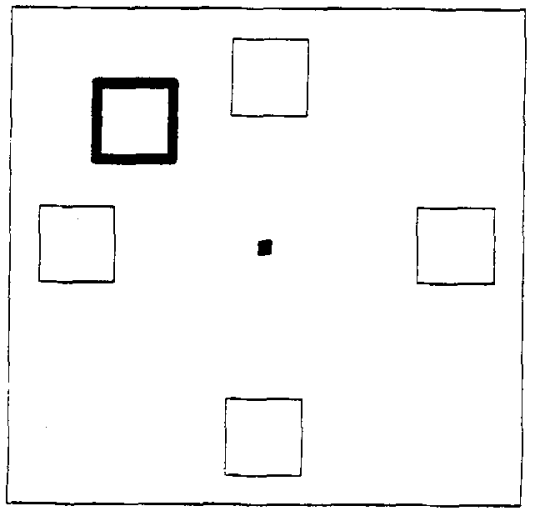

Figure 1. Examples of target and distractor displays for Experiment 1. On the computer display, black characters were bright white, and the open character was red.

onset or by color that could appear in one of four boxes (Figure 1). These target displays were paired with distractor displays 1 that appeared $150 \mathrm{msec}$ prior to the target display and that consisted either of the abrupt brightening of an existing box (i.e., an "old-object" distractor) or the abrupt appearance of a box in a previously blank location (i.e., a "new-object" distractor). Unlike the experiments of Theeuwes $(1994,1996)$, this experiment systematically varied the location of the distractor so that it appeared at the target location on some trials and at nontarget location on other trials. This manipulation ensured that any distractor effects could be attributed to shifts in the spatial distribution of attention, independent of any possible filtering costs. In addition, to ensure that any distractor location effects would reflect involuntary shifts of attention, the distractor was no more likely to appear at the target location than at nontarget locations. Empirically, attentional capture was defined as a significant cost in RTs for dis- tractors appearing at a nontarget location relative to distractors appearing at the target location.

Given the results of Folk et al. (1992), we expected that when the subject was searching for an onset target, the distractor should produce evidence of attentional capture regardless of whether it was a new object or not, because both distractor and target are defined by a luminance change. Specifically, when both "old-object" and "newobject" distractors appear at a nontarget location, RTs should be significantly longer than when distractors appear at the target location. We also expected that when the subject was searching for a color target, an old-object distractor should not produce any evidence of capture (i.e., there should be no difference in RTs for old-object distractors appearing at target vs. nontarget locations). The critical condition is when color targets are preceded by a new-object distractor. If, as suggested by the results of Theeuwes (1994), new objects can override a top-down 
attentional set for color, then even when the subject is searching for a color target, new-object distractors should produce significantly longer RTs than old-object distractors appearing at either target or nontarget locations. If, however, capture by new objects is subject to top-down attentional set, then when the subject is searching for color targets, distractor location should have no effect on RTs, regardless of whether the distractor is a new object or not.

\section{EXPERIMENT 1}

\section{Method}

Subjects. Twelve subjects from Villanova University, ranging in age from 18 to 20 years, participated in partial fulfillment of a course requirement. Subjects were tested for normal or corrected-tonormal binocular near visual acuity ( $20 / 30$ or better from a distance of $14 \mathrm{in.)}$ and normal color vision using a Titmus II vision tester.

Apparatus. Stimuli were presented on a Princeton Graphics Systems Ultrasync monitor, controlled by a Zenith 286 microcomputer equipped with a Sigma Design, Color $400(680 \times 400)$ graphics board. Subjects viewed the monitor from a distance of $50 \mathrm{~cm}$ through lenseless goggles attached to a porthole on the front of a viewing box. The inside of the box was painted black, and all but the screen of the monitor was occluded by a black baffle.

Stimuli. Stimuli consisted of three basic displays: fixation display, distractor display, and target display. In the fixation display, the central fixation square $\left(.34^{\circ} \times .34^{\circ}\right.$ visual angle) was surrounded by four peripheral boxes $\left(1.15^{\circ} \times 1.15^{\circ}\right)$ placed above, below, to the left, and to the right of fixation on an imaginary circle with a diameter of $8.2^{\circ}$. All boxes were light gray (IBM color designation 7) against the black background of the CRT screen.

Two types of distractor displays were used (Figure 1). New-object distractor displays consisted of the fixation display with an additional box (i.e., the distractor) placed in one of four positions on the imaginary circle (upper right, lower right, upper left, or lower left). This distractor appeared in bright white (color 15). Old-object distractor displays consisted of the fixation display with one of the four peripheral boxes (i.e., the distractor) appearing in bright white.

Distractor displays occurred in the context of two types of target display (Figure 1). Onset-target displays consisted of the fixation display with the addition of an " $X$ " or "=" (i.e., the target) in one the peripheral boxes. The target subtended approximately $.57^{\circ}$ of visual angle in height and width and appeared in bright white (color 15). Color-target displays contained an "X" or "=" in each of the four boxes, but one character (i.e., the target) appeared in red (color 12) and the remaining three in white (color 15).

Design. The two target types (onset vs. color) were varied within subjects but were presented in units of three blocks of 40 trials, with order of units balanced across subjects. The two target types were crossed with three distractor types, which were varied within blocks. Distractor types were defined by their spatial relationship to the target, as well as by their object status: In each 40 -trial block, 8 trials were same location/old object, 16 were different location/old object, and 16 were different location/new object. Within each of the latter two conditions, the specific location of the distractor was assigned randomly. Note that with the distractor appearing at the target location on only one fifth of the trials, there is no incentive for the subject to voluntarily shift attention to the location of the distractor. Thus, any effect of distractor location relative to target location can be attributed to involuntary shifts of spatial attention.

Within each block, each target character (i.e., "X" or =) appeared equally often in each of the four possible locations. In the color-target condition, the identity of the characters that appeared in the three nontarget locations was chosen randomly on each trial.

Procedure. Subjects participated in one 60 -min session. The experimenter first tested each subject for normal or corrected-to-normal visual acuity and color vision. Written and oral descriptions of the stimuli and procedures were then provided to familiarize subjects with the task. Speed and accuracy of response were both emphasized, as was the maintenance of fixation on the central square. Subjects were also given full information regarding the relationship (or lack thereof) between the distractor and target locations and were encouraged to "ignore the distractor if possible."

Each block of trials began with a message on the computer screen indicating which of the two target conditions would occur in that block (i.e., onset or color). Subjects pressed the "Enter" key to start the block. At the end of a block, a "rest" message appeared on the display screen.

The trial sequence was as follows. First, the central fixation square and four surrounding boxes appeared for $500 \mathrm{msec}$. The fixation square then blinked off for $100 \mathrm{msec}$ then back on for a randomly varying foreperiod of $1,000,1,100,1,200,1,300$, or $1,400 \mathrm{msec}$. The distractor display then appeared for $50 \mathrm{msec}$, followed by the fixation display for $100 \mathrm{msec}$. The target display then appeared for $50 \mathrm{msec}$, followed once again by the fixation display. The next trial sequence was initiated $1,000 \mathrm{msec}$ after a response was made. Phenomenally, the four display boxes and the fixation cross appeared to remain on the CRT screen for the duration of each trial, as well as the intertrial interval. The stimulus onset asynchrony (SOA) between cue and target was $150 \mathrm{msec}$, making contamination of RTs by eye movements unlikely.

Responses consisted of a press of the "." or " 0 " key on the numeric keypad of the keyboard for " $X$ " and "=" targets, respectively (the keys were appropriately labeled). The " $X$ " response was assigned to the right index finger and the "=" response to the left index finger. RT was measured from the onset of the target display. If a response was not initiated within $1,500 \mathrm{msec}$, an error was scored and the next trial sequence initiated. Incorrect responses elicited a $500-\mathrm{msec}, 1000-\mathrm{Hz}$ computer tone, and were followed by a "buffer" trial with parameters drawn randomly from the set for that block. RTs for error and buffer trials were not included in the data analysis.

\section{Results}

Mean RTs and error rates as a function of distractor condition for each target condition are shown in Figure 2. A within-subjects analysis of variance (ANOVA) with distractor condition and target condition as variables yielded a significant main effect of target condition $[F(1,11)=$ $30.21, p<.001]$, with slower overall RTs to color targets than to onset targets. This result is consistent with previous work (Folk et al., 1992) and presumably reflects an increased perceptual load in the color-target condition relative to the onset-target condition. There was also a significant main effect of distractor condition $[F(2,22)=8.37$, $p<.01]$, but as is evident in the figure, this effect was qualified by a significant interaction with target condition $[F(2,22)=7.30, p<.01]$. Simple effects analyses confirmed that distractor condition significantly affected search for onset targets $[F(2,22)=19.25, p<.001]$, but not search for color targets $[F(2,33)=1.93, p>.05]$. A Tukey test (alpha $=.05$ ) on the means in the onset-target condition established that responses were significantly faster in the same-location condition than in either of the different-location conditions, which did not differ significantly from each other.

Overall error rate was $3.5 \%$. An ANOVA yielded a significant main effect of distractor condition $[F(2,22)=3.65$, $p<.05]$. No other effects were significant. 


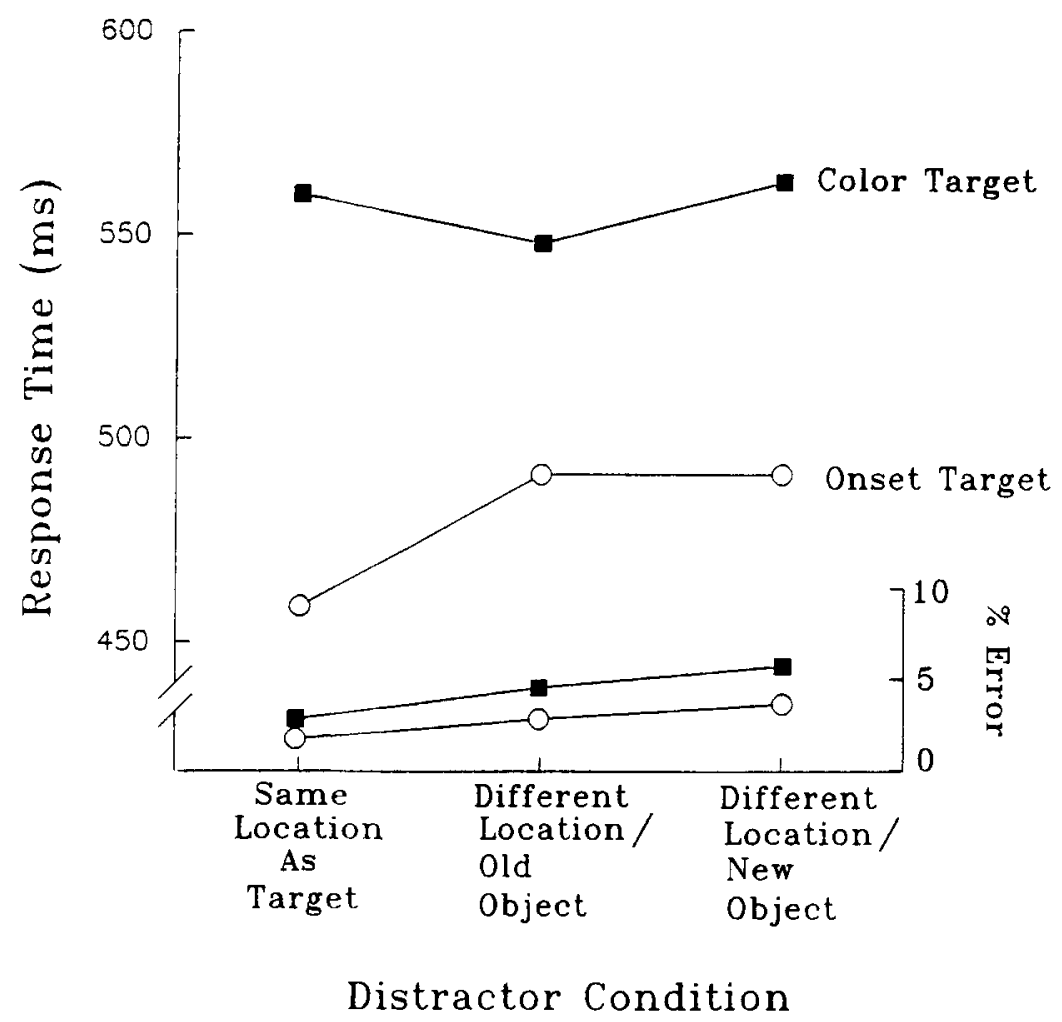

Figure 2. Average mean response times and error rates for distractor conditions as a function of target condition in Experiment 1.

\section{Discussion}

The results of the present experiment replicate those of Folk et al. (1992) in that a luminance change at an existing location produced evidence of attentional capture when the subject was searching for an abruptly onset target, but not when the subject was searching for a target defined by color. This result establishes that the abrupt luminance change distractor is capable of capturing attention, but that such capture is contingent on a top-down attentional set for luminance change.

More importantly, however, the present results suggest that the appearance of a new object cannot override a topdown set for color. Specifically, in the color-target condition, RTs for the different-location/new-object condition did not differ significantly from either of the other two distractor conditions. This suggests that when set for color, the appearance of the new object had no effect on the distribution of spatial attention.

\section{EXPERIMENT 2}

The results of Experiment 1 suggest that new objects cannot override a top-down attention set for color. One might argue, however, that the appearance of the new object was so brief (i.e., $50 \mathrm{msec}$ ) that there was insufficient time for an "object file" to become established (see Yantis \& Gibson, 1994). Alternatively, perhaps attention was drawn to the new object, but its rapid disappearance allowed attention to recover before the target display appeared. To address these alternative accounts, the first experiment was repeated, but with the new-object distractor remaining on the display along with the existing boxes until the subject made a response.

\section{Method}

Subjects. Twelve new subjects were recruited from the same subject pool and under the same conditions as in Experiment 1. All were tested for normal or corrected-to-normal binocular near visual acuity and normal color vision.

Apparatus and Stimuli. Stimuli and apparatus were identical to those used in Experiment 1, except that the distractor remained on the display from distractor onset until subject response. This meant that on trials in which an existing box was brightened, the box remained bright until response. Similarly, on new-object trials, a new bright box appeared and remained until response.

Design. The design and procedure was identical to those used in Experiment 1.

\section{Results}

Mean RTs and error rates as a function of distractor condition for each target condition are shown in Figure 3. As in the first experiment, a within-subjects ANOVA with distractor condition and target condition as variables yielded a significant main effect of target condition $[F(1,11)=21.90, p<.001]$, with slower overall RTs to color targets than to onset targets. There was also a signif- 


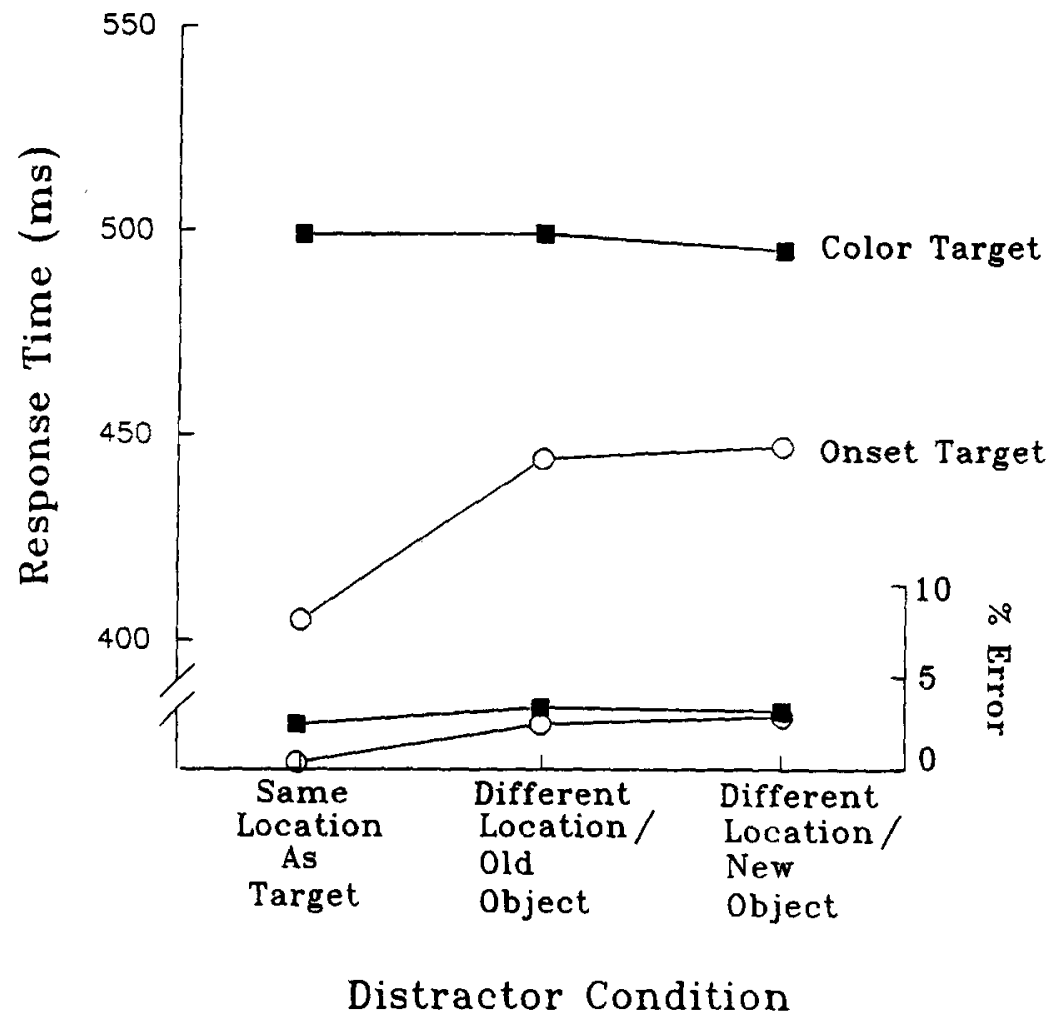

Figure 3. Average mean response times and error rates for distractor conditions as a function of target condition in Experiment 2.

icant main effect of distractor condition $[F(2,22)=8.97$, $p<.01]$, but again this variable entered into a significant interaction with target condition $[F(2,22)=9.06, p<$ $.01]$. Simple effects yielded a significant effect of distractor condition for onset targets $[F(2,22)=31.68, p<$ $.001]$, but not for color targets $(F<1)$. A Tukey test (alpha $=.05$ ) on the means in the onset-target condition revealed that $\mathrm{RT}$ s in the same-location condition were significantly faster than in either of the different-location conditions, which did not differ significantly from each other.

Overall error rate averaged $2.4 \%$. An ANOVA yielded a significant main effect of target type $[F(1,11)=7.37$, $p<.05]$. No other effects were significant.

\section{Discussion}

The results of this experiment are nearly identical to those of Experiment 1. Even when the distractor remained on the display until response, there was no evidence of attentional capture when targets were defined by color, regardless of the object status of the distractor.

\section{EXPERIMENT 3}

The results of the second experiment effectively rule out the possibility that the failure of a new perceptual object to override an attentional set for color is a function of brief presentation time. However, it does not necessarily rule out the possibility that new objects actually do cap- ture attention when set for a color target, but subjects are able to quickly recover from capture when they realize the new object is not the defining color. Folk and Remington (1998) have recently provided evidence against such interpretations of contingent orienting by reducing the SOA between distractor and target displays. They reasoned that on the recovery account, capture should be apparent at short SOAs, before subjects have had a chance to reorient spatial attention. Their results, however, indicated that even at short $(50-\mathrm{msec})$ SOAs, there was no evidence of attentional capture when the distractor color did not match the target color.

In the third experiment, we tested the recovery account in the context of capture by new perceptual objects by reducing the SOA between distractor and target displays from 150 to $50 \mathrm{msec}$. If, in the first two experiments, subjects were captured by new perceptual objects but were able to recover quickly when the target was defined by color, then this capture should be apparent at the short SOA used in the present experiment.

\section{Method}

Subjects. Twelve new subjects participated under the same conditions and meeting the same inclusion criteria as in the previous two experiments.

Apparatus and Stimuli. Stimuli and apparatus were identical to those used in Experiment 2.

Design. The design and procedure were identical to those used in Experiment 2, with the exception that the time between the onset 


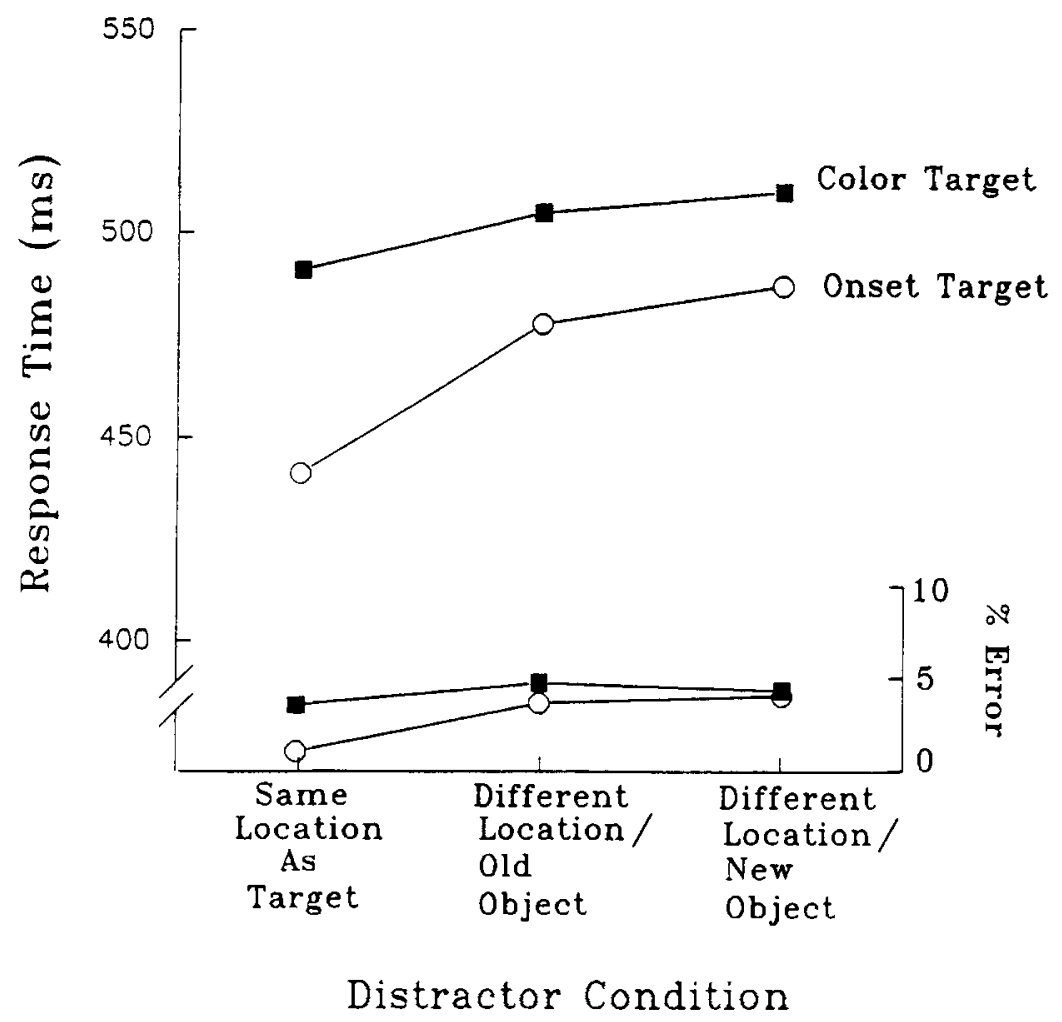

Figure 4. Average mean response times and error rates for distractor conditions as a function of target condition in Experiment 3.

of the distractor display and the onset of the target display was reduced to $50 \mathrm{msec}$.

\section{Results}

Mean RTs and error rates as a function of distractor condition for each target condition are shown in Figure 4. A within-subjects ANOVA yielded a significant main effect of target condition $[F(1,11)=5.58, p<.05]$ and distractor condition $[F(2,22)=24.07, p<.001]$, as well as a significant interaction $[F(2,22)=3.97, p<.05]$. Simple effects yielded a significant effect of distractor condition for onset targets $[F(2,22)=30.40, p<.001]$, but not for color targets $[F(2,22)=2.93, p>.05]$. As in the previous experiments, for the onset-target condition, means for same location trials were significantly faster than in either of the different-location conditions, which did not differ significantly from each other.

Overall error rate averaged $3.5 \%$. An ANOVA yielded a significant main effect of distractor condition $[F(2,22)=$ $4.95, p<.05]$. No other effects were significant.

\section{Discussion}

The pattern of results is identical to that found in the previous two experiments. When the target was defined by color, the distractor produced no evidence of capture regardless of object status. The fact that this pattern obtains even at a relatively short SOA suggests that the apparent lack of capture by new objects in the previous experiments does not simply reflect rapid recovery.

One could still argue, however, that the present experiment is not definitive, because recovery could take place within $50 \mathrm{msec}$. In fact, there was a small trend toward a distractor effect in the color condition, in that RTs in the same-location condition were slightly faster than in the different-location condition. Perhaps this trend reflects the "tail end" of recovery effects. One might also argue that although the measured SOA was $50 \mathrm{msec}$, the functional SOA in the color-target condition might actually have been longer. In all three experiments, overall RTs for the color-target condition were higher than those for the onsettarget condition, presumably reflecting the additional time associated with finding the color target. Perhaps this delay in finding the target lengthens the effective SOA, so that additional time is provided for recovery from capture.

One way of providing a stronger test of the recovery account is to reduce SOA even further, perhaps converging finally on the simultaneous presentation used by Theeuwes (1994). There are several complications associated with such a manipulation, however. First, there is evidence that presenting the distractor simultaneous with the target display produces masking of the target by the distractor when they appear at the same location (Folk et al., 1992, Experiment 4), confounding any attentional effects. More importantly, as the interval between distractor and 


\section{Onset Target}

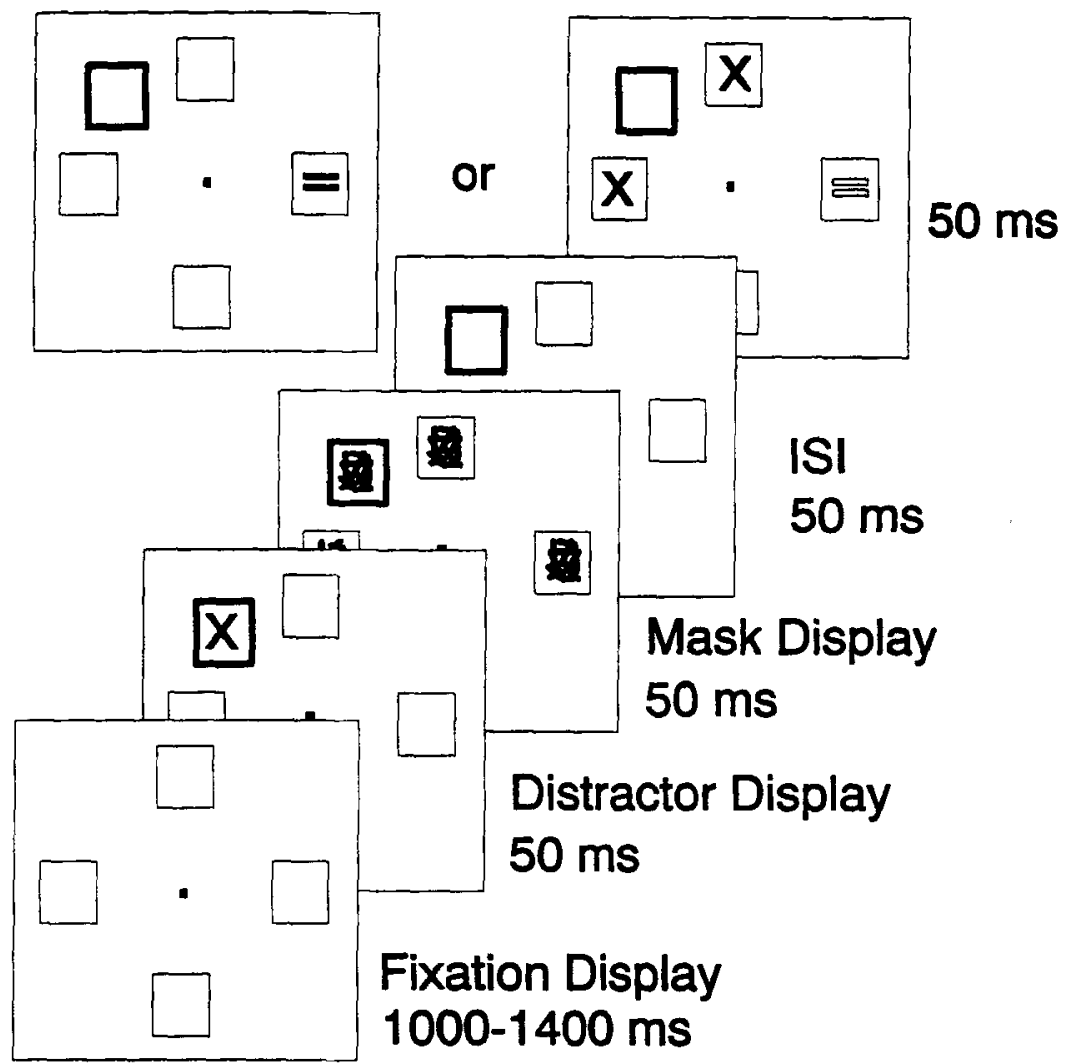

Figure 5. Examples of displays and sequence of events for Experiment 4.

target is reduced, displays in which the distractor and target appear at the same location may be encoded in a fundamentally different way than displays in which the distractor and target appear at different locations. Specifically, when the distractor and target appear simultaneously at the same location, they may be encoded as parts of a single object or event. When they appear simultaneously at different locations, they may be encoded as two distinct objects or events. Thus, any increase in RT in the different-location condition may reflect a filtering cost for "two-object displays" relative to "one-object displays." In other words, with simultaneous presentation or even a very short SOA, it is difficult to determine whether any obtained distractor effects are due to differential filtering costs or involuntary shifts of spatial attention. Note that this ambiguity is obviated at longer SOAs because as distractor and target are separated in time, they are more likely to be encoded as distinct objects or events, even if they occur at the same location.

\section{EXPERIMENT 4}

As an alternate means of testing the recovery account, we replicated Experiment 2, but varied the "compatibility" of the distractor relative to the target character. Specifi- cally, we used the same distractors as in the previous experiments, except that a white "X" or "=" (i.e., the "distractor character," see Figure 5) appeared simultaneous with and inside the distractor box. This display was followed by a pattern mask, which was in turn followed by the presentation of the onset or color target displays used in Experiments 1-3. The pattern mask following the distractor character was intended to equate each location and condition with respect to forward masking of the subsequent target character. The critical aspect of the design is that the compatibility of the distractor character and target character was systematically varied so that on half the trials in each condition the distractor character matched the target character, and on the other half it was the opposite character from the target.

The design is based on the assumption that if attention is allocated to the location of the distractor character, its identity will be processed (Remington, Folk, \& McClean, 1999; Shih \& Sperling, 1996). Given this assumption, if attention is captured by the distractor, then a compatibility effect should be apparent, with longer RTs when the distractor character is incompatible with the target character than when it is compatible. More importantly, this compatibility effect should be apparent regardless of whether spatial attention subsequently "recovers" (disen- 
gages from the distractor) or not. In short, if the recovery account holds, then compatibility effects should obtain in the color-target condition as well as in the onsettarget condition. If, on the other hand, spatial attention is drawn to the distractors when the subject is looking for an onset target but not when the subject is looking for a color target, then one might expect a compatibility effect in the onset-target condition but not in the color-target condition.

\section{Method}

Subjects. Eighteen new subjects participated under the same conditions and meeting the same inclusion criteria as in the previous experiments.

Apparatus and Stimuli. Stimuli and apparatus were identical to those used in Experiment 2, with the following exceptions. First, the distractor displays contained a distractor character appearing inside the distractor box. This character was a bright white (color 15) "X" or "=" of the same dimensions as the target characters. Second, a mask display was introduced after presentation of the distractor display. The mask display was identical to the distractor display for a given trial, except that all boxes (including the new-object box if present) contained a pattern mask made up of short, vertical, horizontal, and diagonal line segments pseudorandomly distributed in an imaginary square subtending $.6^{\circ} \times .6^{\circ}$ of visual angle.

Design and Procedure. The design was identical to that used in Experiment 2 except that the number of trials per block was increased to 80 , and on half the trials in each distractor and target condition the distractor character was compatible with the target character, while on the other half it was incompatible. The procedure was also identical with the exception of the sequence of events on a trial (Figure 5). Specifically, the distractor display appeared for $50 \mathrm{msec}$ and was immediately followed by the mask display for $50 \mathrm{msec}$. The masks were then removed for $50 \mathrm{msec}$ and the target display presented for $50 \mathrm{msec}$. In all other respects, the sequence of events was identical to that used in Experiment 2.

\section{Results}

Mean RTs as a function of distractor condition, compatibility, and target condition are shown in Figure 6, with corresponding error rates shown in Figure 7. A withinsubjects ANOVA on RTs was conducted with target condition, distractor condition, and compatibility as factors. Unlike the RTs in the previous experiments, RTs in the onset-target condition were significantly longer overall than those in the color-target condition $[F(1,17)=11.15$, $p<.01]$ for the main effect. Compatibility also produced a significant main effect $[F(1,17)=22.89, p<.001]$, with incompatible distractors resulting in longer RTs overall than compatible distractors. More importantly, there was a significant interaction between compatibility and target condition $[F(1,17)=16.85, p<.001]$. The mean compatibility effect was $84 \mathrm{msec}$ in the onset-target condition and $1 \mathrm{msec}$ in the color-target condition. Simple effects analyses confirmed that the compatibility effect was significant in the onset-target condition $[F(1,17)=21.07, p<.001]$, but not in the color-target condition $(F<1)$. No other main effects or interactions were significant.

Error rates were much higher than in the previous experiments, averaging $12.8 \%$ overall. As is evident in Figure 7 , however, the overall high error rates were driven primarily by incompatible trials in the onset-target condition. An ANOVA yielded a significant main effect of target condition $[F(1,17)=31.80, p<.0001]$ and distractor compatibility $[F(1,17)=57.67, p<.0001]$. As with RTs, there was also a significant interaction between these variables $[F(1,17)=27.18, p<.0001]$. The compatibility effect was $18 \%$ in the onset-target condition and $1 \%$ in the color-target condition. Finally, target condition and compatibility also entered into a significant three-way interaction with distractor location $[F(2,34)=7.51, p<.01]$. To explore this interaction, simple interaction comparisons were performed for each target condition. For onset targets, compatibility produced a significant main effect $[F(1,17)=42.33, p<.0001]$ and entered into a significant interaction with distractor location $[F(2,34)=5.74, p<$ .011 . For color targets, neither the main effects nor the interaction were significant.

\section{Discussion}

The results of this experiment are quite clear. When the subject was searching for an onset target, the presence of the distractor character produced an overall disruption in performance (relative to performance for onset targets in the previous three experiments) as well as large compatibility effects, in both RTs and error rates. In contrast, when the subject was searching for a color target, the presence and identity of the distractor character had virtually no effect on RT or error rate. This pattern is consistent with the claim that spatial attention is allocated to the location of the distractor in the onset-target condition but not in the color-target condition. The results are inconsistent with the claim that attention is captured in the color-target condition but recovers before target selection. If spatial attention was involuntarily shifted to the distractor in the color-target condition, a compatibility effect should have been apparent regardless of whether attention subsequently recovered or not.

The substantial effect of the presence and identity of the distractor character in the onset-target condition provides strong evidence that spatial attention was allocated to the distractor. It is interesting to note, however, that there were no consistent or reliable effects of distractor location relative to the target location, as there had been in the previous experiments. There are a several possible reasons for the lack of consistent distractor location effects in the present experiment. First, the abrupt onset of masks at all locations following the distractor display may serve to redistribute spatial attention in unpredictable ways, thereby reducing the impact of the spatial location of the distractor on processing of the subsequent target. Second, the processing of the distractor character itself may affect the distribution of spatial attention in unpredictable ways. For example, when spatial attention engages the distractor character and identity processing begins, perhaps spatial attention is "turned off" or "reset" in a way that does not occur when a distractor character is not present (as in the first three experiments). Finally, the processing of the distractor character may simply be so disruptive that the in- 


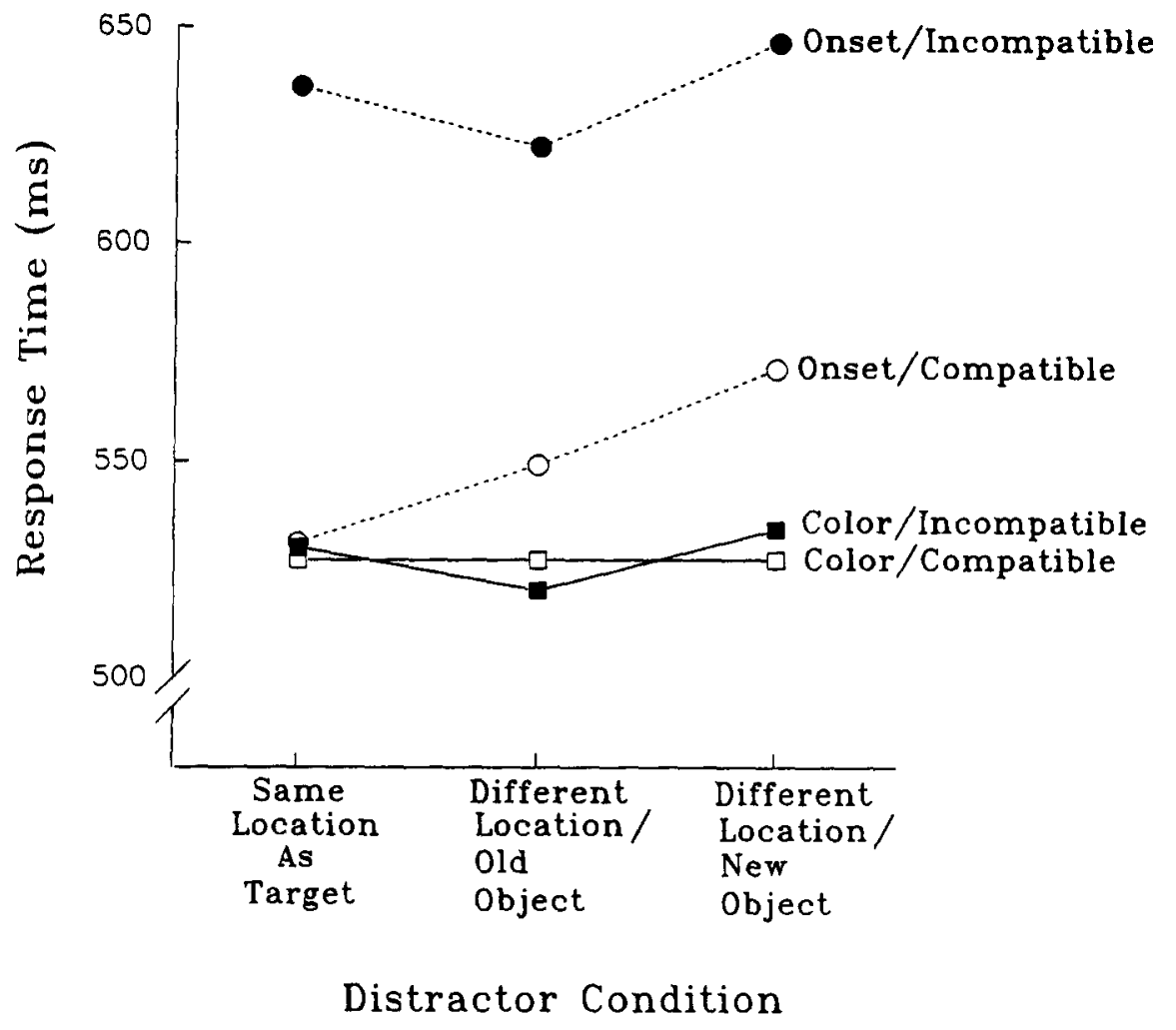

Figure 6. Average mean response times for distractor conditions as a function of compatibility and target condition in Experiment 4.

creased variability in performance makes it difficult to detect the more subtle spatial effects. The large increase in overall RTs, error rates, and variability for onset targets relative to those in the previous experiments is certainly consistent with the latter possibility. It is important to note, however, that overall RTs, error rates, and variability for color targets were quite consistent with those found in the previous experiments.

Given these possibilities, we argue that the lack of a statistically reliable distractor condition effect does not imply that spatial attention was not captured in the onset-target condition. Indeed, we argue that the presence of the identity compatibility effect in the present experiment, combined with the clear evidence of a spatial compatibility effect in the previous experiments, provides converging evidence that when the subject is searching for an onset target, spatial attention is allocated to the distractor location regardless of whether the distractor consists of the brightening of an existing object or the appearance of a new object. Moreover, the lack of either identity or spatial compatibility effects when the subject is searching for color targets provides strong evidence that abrupt onset or new-object distractors cannot override an attentional set for color to produce attentional capture.

\section{EXPERIMENT 5}

The lack of a compatibility effect in the color condition of Experiment 4 was interpreted as evidence that abrupt onsets and new objects cannot override an attentional set for color. This interpretation is based on the assumption that the allocation of attention to a distractor results in the mandatory processing of the distractor character's identity, and should thus have produced a compatibility effect. One might argue, however, that attention was allocated to the distractor even in the color-target condition, but that because the distractor character was a different color (white) from the target (red), it was "filtered out" on the basis of color before its identity was processed. On this account, attention is captured by the distractor, the distractor character's identity is filtered out, and then attention is reoriented so that by the time the target display appears, there are no detectable effects of either the location or identity of the distractor.

Given existing evidence that color filtering is relatively ineffective (e.g., the classic Stroop effect; Remington et al., 1999; Shih \& Sperling, 1996), the filtering of distractor identity in Experiment 4 seems highly unlikely. Nonetheless, to rule out this possibility, an additional control experiment was conducted. We replicated the colortarget condition of Experiment 4 but modified the distractor display so that the distractor consisted of a red box among white boxes. Our previous research has shown that a red distractor produces attentional capture when subjects are set to respond to a red target character (Folk \& Remington, 1998; Folk et al., 1992; Folk et al., 1994). Thus, we expected to find an effect of distractor location consistent with the capture of spatial attention. The critical as- 


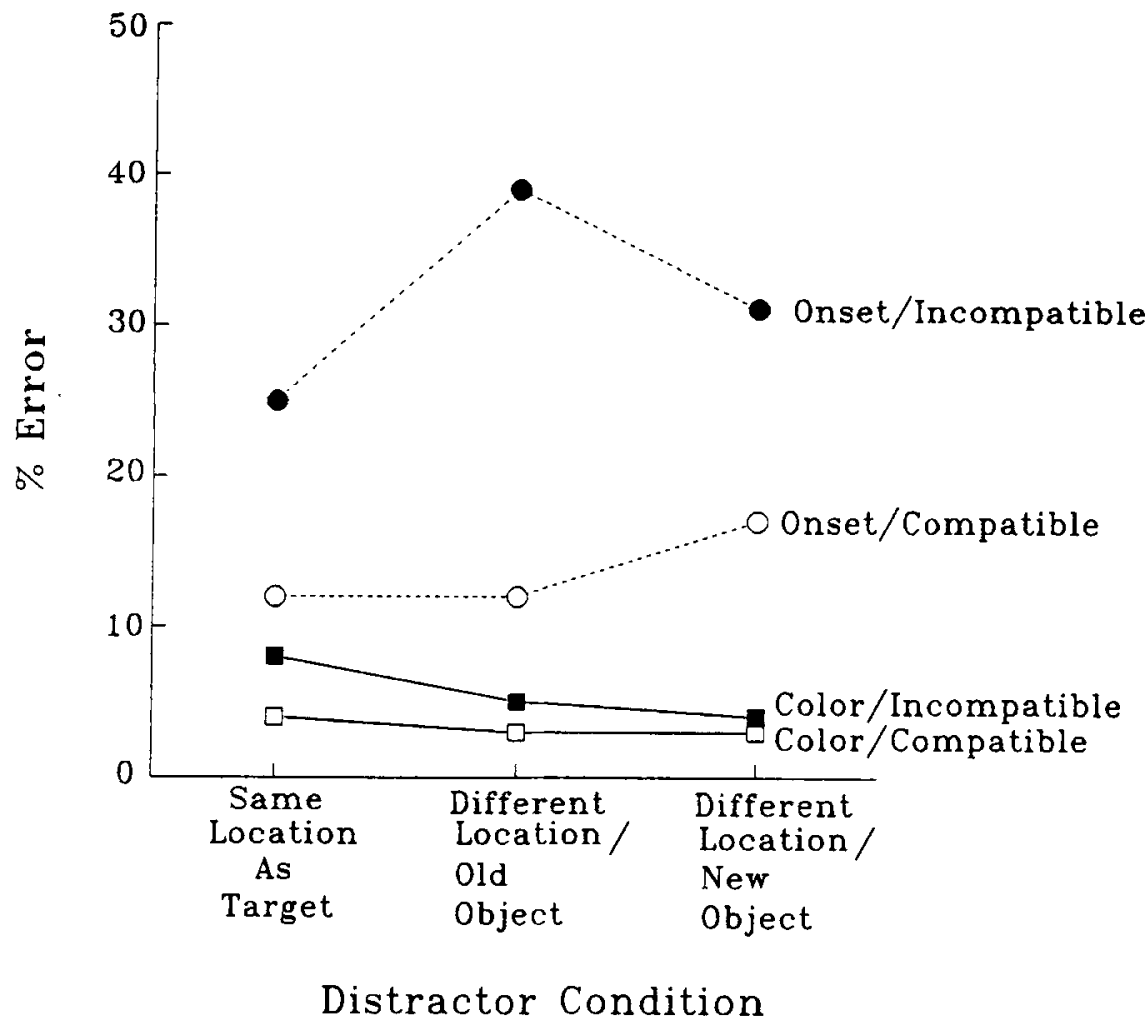

Figure 7. Average mean error rates for distractor conditions as a function of compatibility and target condition in Experiment 4.

pect of the design was that the red distractor box contained a white distractor character identical to that used in Experiment 4 . If subjects are able to filter out the identity of the distractor character on the basis of color, then there should be no compatibility effect because the distractor character is white and the target character is red (as in Experiment 4 ). The presence of a compatibility effect, on the other hand, would indicate that once attention has been allocated to the distractor location, filtering identity on the basis of color is not possible.

\section{Method}

Subjects. Ten new subjects participated under the same conditions and meeting the same inclusion criteria as in the previous experiments.

Apparatus and Stimuli. The apparatus was identical to that used in Experiment 4. The stimuli were identical to those used in the color-target condition of Experiment 4, with one exception. In the distractor display, all boxes except one appeared in bright white (color 15). One box (i.e., the distractor) appeared in red (color 12).

Design and Procedure. The design and procedure were identical to those used in the color-target condition of Experiment 4.

\section{Results}

Mean RTs (error rates) for the same-location, oldobject, and new-object conditions were $471(.019), 520$ (.033), and $515(.014)$, respectively, for compatible trials, and $527(.063), 532(.034)$, and $543(.041)$, respectively, for incompatible trials. A within-subjects ANOVA on RTs yielded a significant main effect of distractor condition $[F(2,18)=10.19, p<.01]$, a significant 50 -msec main effect of compatibility $[F(1,9)=14.22, p<.01]$, and a significant interaction $[F(2,18)=8.96, p<.01]$. A simple effects analysis yielded significant compatibility effects in the same-location condition $[F(1,9)=18.00, p<.01]$ and the new-object condition $[F(1,9)=8.25, p<.05]$. The compatibility effect was not reliable in the the old-object condition, however $[F(1,9)=2.53, p>.05]$. An ANOVA of error rates yielded a significant main effect of compatibility $[F(1,9)=6.99, p<.05]$. No other effects were significant.

\section{Discussion}

Consistent with our previous research (e.g., Folk et al., 1992), when the subject was searching for a red target, the red distractor produced significant location effects consistent with attentional capture. More importantly, the white distractor character appearing inside the red distractor box produced a significant compatibility effect in both RT and error rate. These results show that when attention is allocated to the distractor, subjects are unable to filter out the identity of the white distractor character. Thus, the lack of compatibility effects in the color condition of Experiment 4 cannot be attributed to filtering the distractor character on the basis of color. 


\section{GENERAL DISCUSSION}

According to the contingent orienting hypothesis, the allocation of spatial attention is ultimately contingent on the top-down attentional set of the observer (Folk et al., 1992). This hypothesis is based in part on the ability of the top-down attentional set to modulate spatial capture by abrupt luminance changes to existing objects. The present experiments extend this theoretical account to encompass the effects of new objects. First, we found that both oldobject and new-object distractors produced evidence of capture when subjects were searching for an onset target. Consistent with contingent involuntary orienting, this result shows that when subjects are set to respond to abrupt changes in luminance, irrelevant abrupt luminance changes capture attention, whether they signal the appearance of a new object or not.

More importantly, when subjects were searching for a target defined by color (i.e., in the presence of an attentional set for color), neither abrupt onsets nor new objects produced evidence of attentional capture. This pattern was evident when the distractor remained on the screen until response, ruling out the possibility that the brief appearance of a new object is unable to elicit or sustain an involuntary attention shift. Moreover, Experiments 3-5 provide converging evidence that when subjects are set for a color target, the apparent lack of capture by abrupt onset or new-object distractors cannot be accounted for by rapid recovery from capture. Specifically, onset and new-object distractors produced no evidence of capture at short SOAs, and they also produced no compatibility effect of a distractor character appearing at the distractor location. The fact that compatibility effects were obtained when the target character and distractor box shared the same finding property (even if the distractor character was a different color than the target) establishes that had attention been captured, it would have been detectable. In short, the results are consistent with contingent involuntary orienting, in that there was no evidence of attentional capture when the distractors did not share the defining property of the target, regardless of the distractors' object status. Put another way, there is no evidence that new objects are able to override attentional control settings.

One might argue, however, that our conclusions are specific to the particular stimuli used. That is, there is always the possibility that other "types" of objects could override attentional control settings. Although we cannot rule out this possibility with the present data, it is difficult, a priori, to postulate properties that would constitute different classes of objects, or to speculate about which classes might be expected to override control settings. The present results do indicate that our stimuli are indeed capable of eliciting attentional capture when they are consistent with attentional control settings. In addition, our stimuli were not significantly different from those used in previous studies of capture by new objects (e.g., Theeuwes, 1994; Yantis \& Gibson, 1994; Yantis \& Hillstrom, 1994). In fact, one might expect the new objects used in the present studies to be particularly salient, in that they not only involved the presentation of an object where none had been before, but they were also always brighter than any other existing objects in the display. Finally, even if other types of objects are eventually shown to override attentional control settings, the present results clearly disconfirm the hypothesis that new objects, in general, produce strongly stimulus-driven shifts; any new model would have to incorporate assumptions about object types and how they interact with top-down attentional set.

One final issue concerns the apparent empirical discrepancy between the present results and those of Theeuwes (1994, 1996). Recall that using a visual search task, Theeuwes (1994) found that the appearance of a newobject distractor produced significant cost in performance relative to a no-distractor baseline even when subjects were searching for a target defined by color. Although this result would appear to be at odds with the present data, we have recently shown that irrelevant distractors can have two distinct effects on performance (Folk \& Remington, 1998). Specifically, we found that an irrelevant distractor, in addition to eliciting involuntary shifts of spatial attention when it is consistent with attentional control settings, can also produce a nonspatial "filtering cost" (Treisman et al., 1983) relative to a no-distractor condition. Moreover, we found that these two effects are dissociable, in that a distractor that does not match attentional control settings and therefore does not elicit a shift of spatial attention, can nonetheless produce a significant nonspatial filtering cost compared with a no-distractor baseline. Thus, the apparent attentional capture by new objects in Theeuwes (1994) may reflect a filtering cost rather than a shift of spatial attention.

If the results of Theeuwes (1994) reflect filtering costs, however, one might not expect the kind of distractor compatibility effects documented by Theeuwes (1996). In that study, the identity of irrelevant distractors produced compatibility effects in RT to the target character. By the same logic as the present Experiments 4 and 5, one might argue that the presence of a compatibility effect confirms that spatial attention was selectively allocated to the distractor location. However, as we have previously argued (Folk \& Remington, 1998), the obtained compatibility effects in the Theeuwes (1996) study may reflect an initial perceptual segregation of the display into two objects (the target and distractor), followed by the parallel processing of target and distractor identity, in much the same way that "low-perceptual-load" displays have been shown to be processed in parallel (Lavie, 1995). In short, without converging evidence for shifts of spatial attention, such as effects of distractor location, it is difficult to unambiguously attribute compatibility effects to the allocation of spatial attention. In the present experiments, we argue that it is just this convergence of distractor location and compatibility effects that provides strong evidence for spatial attentional capture when the target and distractor share the same finding property. Conversely, it is the absence of both effects that provides strong evidence that 
spatial attention is not captured when target and distractor do not share the same finding property. Clearly further research is needed to determine whether the effects documented by Theeuwes $(1994,1996)$ reflect the capture of spatial attention or a fundamentally different form of attentional capture more akin to visual filtering.

In conclusion, the present series of experiments shows that the capture of spatial attention by the appearance of a new perceptual object is contingent on a top-down set for luminance change. New objects captured spatial attention in the presence of an attentional set for abrupt luminance change but not in the presence of an attentional set for color. These results provide further evidence for the strong role of top-down attentional set in the capture of visual spatial attention.

\section{REFERENCES}

Bacon, W. F., \& Egeth, H. E. (1994). Overriding stimulus-driven attentional capture. Perception \& Psychophysics, 55, 485-496.

Folk, C. L., \& Remington, R. W. (1998). Selectivity in distraction by irrelevant featural singletons: Evidence for two forms of attentional capture. Journal of Experimental Psychologv: Human Perception \& Performance, 24, 847-858.

Folk, C. L., Remington, R. W., \& JohnSton, J. C. (1992). Involuntary covert orienting is contingent on attentional control settings. Journal of Experimental Psychology: Human Perception \& Performance, 18, 1030-1044.

Folk, C. L., Remington, R. W., \& Johnston, J. C. (1993). Contingent attentional capture: A reply to Yantis (1993). Journal of Experimental Psvchology: Human Perception \& Performance, 19, 682-685.

Folk, C. L., Remington, R. W., \& Wright, J. H. (1994). The structure of attentional control: Contingent attentional capture by apparent motion, abrupt onset, and color. Journal of Experimental Psychology: Human Perception \& Performance, 20, 317-329.

GiBson, B. (1996). Visual quality and attentional capture: A challenge to the special role of abrupt onsets. Journal of Experimental Psychology: Human Perception \& Performance, 22, 1496-1504.

GibSON, B., \& KelSEy, E. (1998). Stimulus-driven attentional capture is contingent on attentional set for display-wide visual features. Journal of Experimental Psychology: Human Perception \& Performance, 24, 699-706.

JuOla, J. F., Koshino, H., \& WARNER, C. B. (1995). Tradeoffs between attentional effects of spatial cues and abrupt onsets. Perception \& Psychophysics, 57, 333-342.

LAVIE, N. (1995). Perceptual load as a necessary condition for selective attention. Journal of Experimental Psychology: Human Perception \& Performance, $21,451-468$.
PoSNer, M. I., \& COHEN, Y. (1984). Components of visual orienting. In H. Bouma \& D. G. Bouwhuis (Eds.), Attention and performance $X$ : Control of language processes (pp. 531-556). Hillsdale, NJ: Erlbaum.

Remington, R. W., Folk, C. L., \& McClean, J. (1999). Stimulus processing following involuntary shifts of viswal attention. Manuscript submitted for publication.

ShIH, S., \& Sperling, G. (1996). Is there feature-based attentional selection in visual search? Journal of Experimental Psychology: Human Perception \& Performance, 22, 758-779.

THEEUWES, J. (1991). Exogenous and endogenous control of attention: The effect of visual onsets and offsets. Perception \& Psychophysics, 49, 83-90.

Theeuwes, J. (1992). Perceptual selectivity for color and form. Perception \& Psychophysics, 51, 599-606.

THEEUWES, J. (1994). Stimulus-driven capture and attentional set: Selective search for color and visual abrupt onsets. Journal of Experimental Psychology: Human Perception \& Performance, 20, 799-806.

TheEuwes, J. (1996). Perceptual selectivity for color and form: On the nature of the interference effect. In A. F. Kramer, M. G. H. Coles, \& G. D. Logan (Eds.), Converging operations in the study of visual selective attention (pp. 297-314). Washington, DC: American Psychological Association.

Treisman, A., Kahneman, D., \& Burkell, J. (1983). Perceptual objects and the cost of filtering. Perception \& Psychophysics, 33, 527-532.

YANTIS, S. (1993). Stimulus-driven attentional capture. Current Directions in Psychological Science, 2, 156-161.

YANTIS, S. (1996). Attentional capture in vision. In A. F. Kramer, M. G. H. Coles, \& G. D. Logan (Eds.), Converging operations in the study of visual selective attention (pp. 45-76). Washington, DC: American Psychological Association.

YANTIS, S., \& GibSON, B. S. (1994). Object continuity in apparent motion and attention. Canadian Journal of Experimental Psychology, 48, 182-204.

YanTIS, S., \& Hillstrom, A. P. (1994). Stimulus-driven attentional capture: Evidence from equiluminant visual objects. Journal of Experimental Psychology: Human Perception \& Performance, 20, 95-107.

YANTIS, S., \& JoNiDES, J. (1984). Abrupt visual onsets and selective attention: Evidence from visual search. Journal of Experimental Psychology: Human Perception \& Performance, 10, 601-621.

YANTIS, S., \& JonidES, J. (1990). Abrupt visual onsets and selective attention: Voluntary versus automatic allocation. Journal of Experimental Psychology: Human Perception \& Performance, 16, 121-134.

\section{NOTE}

1. Note that because the location of the distractor provides no information about the subsequent location of the target, we have avoided the use of the term precue even though the distractor display occurs prior to the target display.

(Manuscript received May 5, 1997; revision accepted for publication April 6, 1998.) 